\title{
Detección de seropositividad VIH en embarazadas: consideraciones bioéticas
}

\author{
MIGUEL KOTTOW(1)
}

\section{INTRODUCCIÓN}

La detección de seropositividad VIH en embarazadas es de capital importancia desde que los cuidados perinatales y el tratamiento antirretroviral permiten reducir la transmisión vertical de $25 \%$ a menos de $2 \%$. La tendencia inicial de implantar programas compulsorios para examinar a toda embarazada está siendo reemplazada por la participación voluntaria, o por el examen de rutina que presupone consentimiento implícito, pero deja margen para que sea omitido cuando la mujer hace valer sus razones para rechazarlo.

Desde los inicios de la reflexión bioética sobre problemas de salud pública quedó planteada la inevitable y conflictiva tensión entre intervenciones colectivas y la autonomía de los individuos. Esta divergencia ya había sido presentada como la 'paradoja preventiva': "Una medida preventiva que trae muchos beneficios a la población, ofrece poco a cada individuo participante" (Rose, 1985). La eventual divergencia entre una medida de bien público y los perjuicios individuales que pudiesen resultar, se ve exacerbada cuando un programa sanitario propone medidas compulsorias que son resistidas por algunos individuos al invocar sus derechos humanos y el respeto de su autonomía. En un clima político donde prima el postulado utilitarista de J.S. Mill cuando pregona que la libertad individual sólo ha de ser limitada si infringe la libertad de otros, se deriva que el único principio ético de validez general es el de no dañar. Las consideraciones bioéticas han de ponderar la proporcionalidad entre beneficios de programas obligatorios y el daño que causen a los disidentes al erosionar su derecho a decisión autónoma.
En el vasto y aún muy polémico campo de reflexión bioética en salud pública, se da con urgente relevancia el enfrentamiento de la salud pública con los múltiples problemas planteados por la pandemia contemporánea del VIH y el Sida, donde se entrecruzan incertidumbres científicas y las solicitaciones de equidad sanitaria. Las peculiaridades epidemiológicas del VIH no encuentran adecuada respuesta en la tradición sanitaria, de donde proviene el 'excepcionalismo epidemiológico' que ha llevado a "radicalmente eximir al Sida de las intervenciones tradicionales de la salud pública" (Burr 1997). La bioética se ha visto envuelta en polémicas que avizoran la diversidad de valores comprometidos, afectando temas tan diversos como confidencialidad, declaración obligatoria, tamizajes, coberturas terapéuticas, ensayos clínicos de vacunas y nuevas terapias, permisividad de paternalismos institucionales o estatales a fin de proteger a poblaciones en riesgo, y muchos otros.

Dentro de la plétora de incertidumbres epidemiológicas y bioéticas relacionadas con el complejo VIH/Sida, se discute desde hace algunos años la efectividad y la legitimidad moral de diversas estrategias para prevenir la transmisión vertical del VIH en embarazadas seropositivas. El tema ha tomado especial relevancia desde que se demostrara fehacientemente que el riesgo de transmisión vertical madre-hijo puede ser reducido de $25 \%$ a menos del $8 \%$, a condición de instituir una terapia oportuna y completa, a iniciarse durante el embarazo y ser mantenida más allá del parto. Si a ello se agrega el parto por cesárea, la transmisión vertical puede bajar a un $2 \%$, sobre todo si se limita el periodo de lactancia materna.

Pregunta frecuentemente formulada es si

(1) Escuela de Salud Pública. Facultad de Medicina. Universidad de Chile. Independencia 939. Santiago. Chile. mkottow@med.uchile.cl 
acaso las mujeres que portan un embarazo deben someterse compulsoriamente a exámenes de sangre a fin de detectar seropositividad, sobre todo en naciones con coberturas médicas precarias y programas deficitarios de control materno-infantil. El debate engarza con el tema más general de la ética de tamizajes cuya justificación depende de una serie de criterios ,incluyendo efectividad y oportunidad para instituir medidas terapéuticas adecuadas (Holland 1993). El reciente anuncio del Ministerio de Salud chileno, de instituir el examen de VIH en forma compulsoria en toda embarazada, acentúa la actualidad del tema y requiere abrir su discusión en el marco de la realidad cultural y sanitaria de nuestro país, en consideración de la experiencia internacional y la extensa reflexión bioética suscitada.

\section{FACTORES EPIDEMIOLÓGICOS}

En la región sub-sahariana se estima una incidencia anual de 600.000 seropositivos congénitos. Con una proyección mundial de 5.000 .000 de niños infectados con el VIH, $90 \%$ de ellos contagiados in útero o durante la lactancia prolongada, se alzaron voces abogando por el examen mandatorio de toda embarazada, a fin de salvar muchas vidas, mejorar campañas de prevención, así como ahorrar recursos a largo plazo a pesar de los altos costos iniciales del tratamiento perinatal y los cuidados terapéuticos consecuentes. Los antecedentes epidemiológicos y la evaluación económica son argumentos empleados para legitimar éticamente la introducción de la pesquisa obligatoria de VIH en embarazadas que viven en regiones de alta prevalencia. En el caso de Botswana, por ejemplo, los datos epidemiológicos justificarían, en opinión de algunos, una intervención fuerte con carácter de compulsoria (Clark 2006).

Un modelo estadístico, basado en experiencias publicadas, estima la prevalencia promedio de infección VIH en mujeres en edad fértil en $0.58 \%$, una proporción lo suficientemente baja para, de por sí, justificar un programa voluntario. La aceptación voluntaria del examen de VIH es tan alta $-98.4 \%$-, que una política compulsiva no aumentaría la proporción de personas reclutadas sino, al contrario, disminuiría la cobertura porque las políticas obligatorias siempre tienen una cuota de rechazo que, aunque fuese baja -estimada en $0.5 \%$, o sea, 5 mujeres de 1.000 sometidas a examen obligatorio rechazarían participar-, marginaría a más personas que el esquema voluntario. La conclusión de este estudio es que "los esfuerzos de educación y alcance para aumentar la aceptación voluntaria de examen VIH durante el embarazo sería la estrategia más efectiva para identificar mujeres seropositivas y reducir la transmisión materno-infantil de VIH" (Nakchbandi et al. 1998).

\section{FACTORES MÉDICO-SOCIALES}

En 2007, WHO/UNAIDS presentó un documento denominado PITC [Provider-initated HIV Testing and Counselling in Health Facilities] que, aplicado al problema de HIV en embarazadas recomienda: precocidad en el diagnóstico, examinar a todas las mujeres en trabajo de parto cuyo estatus HIV no sea conocido, ofrecer atención médica posparto, entregar profilaxis y asesoría en la alimentación del recién nacido, asesoría preventiva a mujeres que son VIH (-), e incentivar a las mujeres para incorporar a sus parejas en la prevención de HIV (Gruskin, et al. 2008). A fin de respetar los derechos humanos de las mujeres, las estrategias de salud pública deben ser basadas en evidencia y abiertamente debatidas, evitando utilizar "estrategias inciertas y potencialmente contraproducentes, incluso aquellas motivadas por genuina desesperación de cara a desafíos ingentes a la salud pública" (Gruskin and Loff 2002). Esta advertencia se dirige al ya mencionado excepcionalismo epidemiológico que desaconseja la aplicación indiscriminada de medidas sanitarias tradicionales, dado el carácter peculiar y aún insuficientemente explorado del complejo VIH/Sida (Burr 1997). En el presente contexto, el excepcionalismo no se refiere tanto a la omisión de medidas epidemiológicas habituales en situaciones epidé- 
micas -aislamiento, seguimiento de contagios, pesquisas compulsorias-, dada su escasa utilidad para controlar la enfermedad, y el alto grado de daños y discriminaciones. Se tiende, en consecuencia, a abandonar los tamizajes masivos y las medidas obligatorias de detección e intervención con consentimiento informado formal, reemplazándolos por el examen de HIV incorporado a la rutina sanitaria de grupos de alto riesgo, en quienes se supone un consentimiento implícito pero revocable a voluntad (Bayer and Fairchild 2006). De este modo, se evita la violación de derechos humanos que producen programas compulsorios, sin por ello sacrificar eficacia epidemiológica.

Las guías de acción propuestas y periódicamente revisadas, difieren sustancialmente según el contexto socioeconómico en que se dan, mas tampoco coinciden para una misma realidad social, puesto que se basan en diferencias de selección de datos y variaciones de interpretación, llevando a estrategias de acción discrepantes (Chou 2011), no pocas veces debidas a la influencia de conflictos de intereses intelectuales o financieros (Guyatt, et al. 2010). Ello no obstante, las políticas sanitarias serán plausibles y aceptables en la medida que consideren informaciones empíricas que, sin ser precisas, orientan sobre el mejor camino a seguir. Las guías de acción son revisadas periódicamente, habiendo evolucionado desde el examen obligatorio avalado por un consentimiento informado documentado, a programas que incorporan la exploración de VIH como procedimiento de rutina que supone un consentimiento implícito pero que puede ser revocado por quienes rechacen el examen. Una modalidad que gana cada vez más adeptos es ofrecer el acceso voluntario al examen de VIH, en el entendido que el conocimiento de su estatus serológico permite a las personas hacer uso de programas preventivos y terapéuticos solventados por recursos públicos, además de adecuar su conducta social para reducir los riesgos de contagio.

En recientes años se ha introducido el examen serológico rápido para mujeres que no han tenido controles obstétricos regulares, lo cual permite en una hora hacer el diagnóstico e instituir terapia de emergencia aun durante el parto (Bulterys, et al. 2004). Se ha sugerido que el diagnóstico rápido sea mandatorio, dada la urgencia de intervenir y porque la mujer, al encontrarse en inminente trabajo de parto, no estaría en condiciones de lucidez para dar su consentimiento informado a una recomendación voluntaria de explorar si acaso es VIH (+). Esta modalidad diagnóstica y terapéutica reduce substancialmente la infección neonatal, aunque es menos segura que el tratamiento completo recomendado por el protocolo ACTG- 076, estimándose especialmente apropiada para poblaciones con atención médica precaria, donde las mujeres no acceden a programas materno-infantiles integrales, sino que acuden por primera vez al centro médico cuando el parto es inminente. En este contexto, es probable que la terapia de emergencia se realice con una sola droga, lo cual es suficiente para proteger al neonato, pero aumenta la posibilidad de resistencias a drogas retrovirales en el futuro terapéutico de la mujer.

\section{ASPECTOS BIOÉTICOS}

Hay consideraciones éticas que cuestionan el empleo de programas obligatorios aunque sólo sea en situaciones de emergencia, por cuanto se viola la autonomía de las personas al menos en dos aspectos: las intervenciones compulsorias no dan lugar a ejercer la autonomía de objetar y rehusar la participación y, en segundo lugar, un programa compulsorio debe dar amplio acceso a los datos y con ello pone en riesgo la confidencialidad. Para evitar los daños de una alta incidencia de niños infectados con VIH, es preciso violentar la autonomía de la embarazada al someterla a examen en ausencia de, o incluso contra, su voluntad, además de obligarla al tratamiento posterior, lo cual a su vez viola el derecho del paciente a rehusar tratamientos [Declaración de Lisboa, presentada por la Asociación Médica Mundial en 1981 y ratificada en 1995). La política sanitaria de muchos países es de informar y promover el examen voluntario de embarazadas, 
desestimando los argumentos de quienes insisten en programas obligatorios para poblaciones con alta prevalencia de infección.

El aspecto más polémico se refiere a la necesidad o inconveniencia de diversas formas de pesquisar seropositividad, ante todo comparando ventajas y desventajas de la obligatoriedad versus la voluntariedad de ser examinado o, alternativamente, de considerar el consentimiento presunto de quienes reciben atención médica que de rutina incluye el examen de HIV. La obligatoriedad sólo se sustentaría si se acompaña de un acceso irrestricto a las mejores medidas preventivas y terapéuticas actualmente en existencia para personas seropositivas asintomáticas y para aquellas cuyo estado inmunitario se deteriora o por la aparición clínica del Sida. Sin embargo, la evidencia empírica, así como el análisis de modelos de decisión, sugieren que la obligatoriedad no ofrece ventajas epidemiológicas y médicas sobre el reclutamiento voluntario cuando se da la situación de una baja prevalencia y una alta disposición a recurrir voluntariamente a los servicios de detección, prevención y tratamiento.

Ante la insistencia de establecer programas de control obligatorio en embarazadas, o de prescindir del consentimiento informado en situaciones de emergencia como lo es el parto de mujeres que no han estado en control obstétrico, es preciso evaluar los aspectos negativos de la obligatoriedad. En más de una ocasión se ha planteado que la obligatoriedad se extendería, en caso necesario, a indicar el parto por cesárea, el tratamiento oral de la madre, la limitación del periodo de lactancia, todas medidas que implican un paternalismo autoritario que desatiende los derechos de autonomía de las mujeres (Bayer, 1994). Existen factores sociales que la mujer podría aducir para rechazar el examen: estigmatización frente a su grupo social, riesgo aumentado de violencia familiar -cosa empíricamente comprobada-. En Malawi, por ejemplo, la mujer reconocida como $\mathrm{VIH}(+)$ es discriminada y acusada de promiscuidad, adulterio y prostitución, sufriendo abandono, ostracismo, violencia y maltratos que se extienden a sus hijos. La revelación pública de la seropositividad puede ocurrir por violaciones o filtraciones de la confidencialidad. Paradójicamente, los programas preventivos de transmisión vertical son delatores al evidenciar controles periódicos y visitas domiciliarias, reducción de la lactancia materna, la presentación de incentivos y la visibilidad de los puestos de atención de estos programas conocidos como PMTCT [prevention of mother-to-child transmission] (Thorsen et al. 2008).

Cuando la mujer no tiene acceso a servicios médicos y éstos a su vez son precarios, es probable que no obtenga los beneficios de medicación permanente y suficiente que un diagnóstico oportuno o de emergencia debiera asegurar. En suma, los costos sociales y la probabilidad de ineficacia terapéutica si durante el parto recibió una terapia que puede crearle futuras resistencias farmacológicas, son todas consideraciones que hacen plausible que algunas mujeres rechazarían indagar su estado VIH, sintiéndose vulneradas en su autonomía si hubiesen sido compulsoriamente examinadas.

Se suscita el dilema ético conocido de enfrentar la protección y los derechos del nonato frente a la autonomía y los derechos de la embarazada o parturienta: ¿puede la mujer ejercer su derecho de autonomía aun cuando su decisión fuese perjudicial para el embrión o feto? ¿Es éticamente requerido que acepte las desventajas de un diagnóstico y tratamiento de urgencia, pero que será del todo, aunque no siempre, ventajoso para el nonato?

Ante la aporía ética de tener que decidir entre alternativas que ambas entrañan daños importantes -para la mujer, para el niño o para ambos-, se sugiere recurrir a la doctrina del mal menor, evaluada según criterios de razonamiento proporcional (McCormick 1989): limitar el daño al mínimo posible, tener certeza de que no hay alternativas menos dañinas, y que los medios a emplear no invalidarán el valor positivo que se busca. Uno de los inconvenientes del proporcionalismo es que, para no ser arbitrario en la ponderación de bien y mal, 
tendría que recurrirse a datos cuantitativos, que en el presente caso se referirían a la prevalencia de HIV/Sida en la población de mujeres fértiles, la certeza terapéutica de diversos regímenes de tratamiento, los costos reales de las diversas alternativas de acción, las posibilidades financieras de organizar programas materno-infantiles integrales, la proporción de mujeres que verían violada su autonomía frente a una política compulsoria.

En otras palabras, en contextos de pobreza e insuficiencia de recursos médicos, el examen y tratamiento mandatorios perjudicarían a la mujer y la privarían de la oportunidad de negarse al procedimiento. La aplicación de exámenes diagnósticos de emergencia durante el trabajo de parto en que la mujer puede no estar en condiciones de otorgar un consentimiento informado válido, ha sido cuestionada por la probabilidad de condenar a la mujer que resulte seropositiva a los riesgos de violencia familiar y ostracismo social. Si se persiste en hacer compulsoria esta política de emergencia, es de temer que más mujeres eviten recurrir a asistencia médica y aumente la desconfianza de quienes no pueden o no quieren entrar en regímenes de vigilancia sanitaria (Smith, et al. 2007). Ponderando estos argumentos, las recomendaciones internacionales toman otro camino: "UNAIDS/WHO no aprueba el examen obligatorio de individuos por motivos de salud pública. El examen voluntario tiene mayores probabilidades de llevar a cambios conductuales que impidan la transmisión del VIH a otros individuos."

\section{DISCUSIÓN}

El complejo VIH/Sida es un problema de gran envergadura para la salud pública de naciones tanto ricas como para las menos desarrolladas, debiendo ser abordado con un substancial acopio de recursos, por lo cual las políticas públicas han de basarse en conocimientos epidemiológicos confiables y con el uso racional de los ingentes recursos necesarios.

La prevalencia de seropositividad al VIH en la "población gestante chilena" es de $0.5 / 1000$, la de mujeres embarazadas VIH (+) alcanza $0.054 \%$ (Ovalle, et al. 2003; Valdés 2002). Según criterios internacionales, estas cifras son tan bajas que no justifican la implantación de programas obligatorios. La alta disposición a participar en programas voluntarios que se registra en diversas culturas, posiblemente también se dé en nuestro país, asunto que al menos debiera ser evaluado antes de proclamar programas autoritarios que, la experiencia lo demuestra, desatienden los derechos humanos y violentan la autonomía de aquellas mujeres que por razones plausibles consideran que se verían perjudicadas en su vida familiar y social. La tendencia de los programas de prevención ha sido de proporcionar una monoterapia de mantención con nevirapine, con los riesgos de resistencias posteriores. Las políticas autoritarias carecen de toda justificación cuando no existe un compromiso explícito de otorgar y garantizar todos los beneficios médicos que la detección de una seropositividad requiere. (Eyakuze, et al. 2008).

Al proclamar públicamente el Ministerio de Salud la implantación de un programa obligatorio de pesquisa de VIH en embarazadas, cabe suponer que ha analizado el problema a fondo y llegado a la improbable conclusión que, contrariamente a la tendencia mundial, los programas compulsorios son más eficaces $\mathrm{y}$ menos perjudiciales que los voluntarios o los rutinarios con consentimiento implícito pero revocable. Aun reconociendo que políticas y programas de salud pública deben respetar las realidades y los contextos donde se aplican, resulta difícil entender que Chile desarrolle políticas públicas que han sido desestimadas tanto en países más pobres como en los más desarrollados. En un régimen democrático, las políticas públicas han de ser precedidas por una revisión acuciosa de la experiencia internacional, y un ponderado análisis de la reflexión bioética, a fin de llegar a propuestas ampliamente acordadas. Es de esperar que el Departamento de Estudios y la asesoría bioética del Minsal ayuden a diseñar una campaña de educación y promoción que permita a la ciudada- 
nía participar en las decisiones que desarrollen las mejores iniciativas e intervenciones de la salud pública a objeto de abordar los problemas del complejo VIH/Sida en forma racional, técnicamente eficaz y moralmente validada.

\section{REFERENCIAS}

1. BAYER, R. Ethical challenges posed by zidovudine treatment to reduce vertical transmission of HIV. N Engl J Med 331(18):1223-5. 1994.

2. BAYER, R., AND A. L. FAIRCHILD. Changing the paradigm for HIV testing--the end of exceptionalism. N Engl J Med 355(7):647-9. 2006.

3. BULTERYS, M., et al. Rapid HIV-1 testing during labor: a multicenter study. JAMA 292(2):219-23. 2004.

4. BURR, CH.The AIDS edxception: privacy vs. punlic health. -the Atlantic Monthly June:57-67. 1997.

5. CLARK, P.A. Mother-to-child transmission of HIV in Botswana: an ethical perpective on mandatory testing. Developing World Bioethics 6:1-12. 2006.

6. CHOU, R. Routine screening for chronic human immunodeficiency virus infection:why don't the guidelines agree. Epidemiologic Reviews 33:7-19. 2011.

7. EYAKUZE, C., et al. From PMTCT to a more comprehensive AIDS response for women: a muchneeded shift. Dev World Bioeth 8(1):33-42. 2008.

8. GRUSKIN, S., S. AHMED, AND L. FERGUSON. Provider-initiated HIV testing and counseling in health facilities--what does this mean for the health and human rights of pregnant women? Dev World Bioeth 8(1):23-32. 2008.
9. GRUSKIN, S., AND B. LOFF. Do human rights have a role in public health work? Lancet 360(9348):1880. 2002.

10. GUYATT, G., et al.The vexing problem of guidelines and conflict of interest: a potential solution. Ann Intern Med 152(11):738-41. 2010.

11. HOLLAND, W. W. Screening: reasons to be cautious. BMJ 306(6887):1222-3. 1993.

12. MCCORMICK, R.A. Reflections on moral dilemmas since Vatican II. Washington, D.C.: Georgetwon University Press. 1989.

13. NAKCHBANDI, I. A., et al. A decision analysis of mandatory compared with voluntary HIV testing in pregnant women. Ann Intern Med 128(9):760-7. 1998.

14. OVALLE, A., et al. [Human immunodeficiency virus infection in pregnant women. The importance of recognizing the infection during pregnancy and risk factors for perinatal transmission]. Rev Med Chil 131(6):633-40. 2003.

15. ROSE, G. Sick individuals and sick populations. International Journal of Epidemiology 14:32-38. 1985

16. SMITH, C. B., et al. Should rapid tests for HIV infection now be mandatory during pregnancy? Global differences in scarcity and a dilemma of technological advance. Dev World Bioeth 7(2):86-103. 2007.

17. THORSEN, V. C., J. SUNDBY, AND F. MARTINSON. Potential initiators of HIV-related stigmatization: ethical and programmatic challenges for PMTCT programs. Dev World Bioeth 8(1):43-50. 2008.

18. VALDÉS, E.R. VIH-Sida y embarazo. Actualización y realidad en Chile. Rev Chil Obstet Ginecol 67:160168. 2002. 